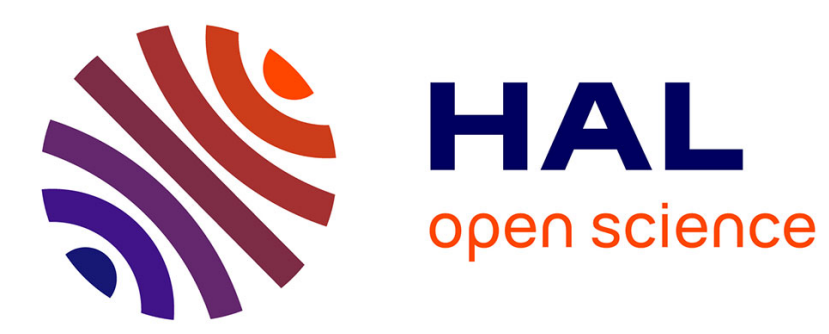

\title{
Influence of the "Ghost Reed" Simplification on the Bifurcation Diagram of a Saxophone Model
} \author{
Tom Colinot, Louis Guillot, Christophe Vergez, Philippe Guillemain,
} Jean-Baptiste Doc, Bruno Cochelin

\section{- To cite this version:}

Tom Colinot, Louis Guillot, Christophe Vergez, Philippe Guillemain, Jean-Baptiste Doc, et al.. Influence of the "Ghost Reed" Simplification on the Bifurcation Diagram of a Saxophone Model. Acta Acustica united with Acustica, 2019, 105 (6), pp.1291-1294. 10.3813/AAA.919409 . hal-02433732

\section{HAL Id: hal-02433732 \\ https://hal.science/hal-02433732}

Submitted on 9 Jan 2020

HAL is a multi-disciplinary open access archive for the deposit and dissemination of scientific research documents, whether they are published or not. The documents may come from teaching and research institutions in France or abroad, or from public or private research centers.
L'archive ouverte pluridisciplinaire HAL, est destinée au dépôt et à la diffusion de documents scientifiques de niveau recherche, publiés ou non, émanant des établissements d'enseignement et de recherche français ou étrangers, des laboratoires publics ou privés. 


\title{
Influence of the "ghost reed" simplification on the bifurcation diagram of a saxophone model
}

\author{
Tom Colinot ${ }^{\mathrm{a}}$, Louis Guillot ${ }^{\mathrm{a}}$, Christophe Vergez $^{\mathrm{a}}$, Philippe Guillemain ${ }^{\mathrm{a}}$, \\ Jean-Baptiste Doc ${ }^{\mathrm{b}}$, Bruno Cochelin ${ }^{\mathrm{a}}$ \\ a Aix Marseille Univ, CNRS, Centrale Marseille, LMA, Marseille, France \\ b Laboratoire de Mécanique des Structures et des Systèmes couplés, Conservatoire National \\ des Arts et Métiers, Paris, France
}

\section{Summary}

2 This paper presents how the bifurcation diagram of a saxophone model is affected by the contact force limiting the displacement of the reed when it strikes the mouthpiece lay. The reed impact is modeled by a nonlinear stiffness and damping activated by contact with the lay. The impact model is compared with the "ghost reed" simplification, where the reed moves through the lay unimpeded. Bifurcation diagrams in both cases are compared, in terms of amplitude of the oscillations and location of the bifurcations, on the solution branches corresponding to the first and second register. The ghost reed simplification has limited influence at low values of the blowing pressure parameter: the diagrams are similar. This is true even for "beating reed" regimes, in which the reed coincides with the lay. The most noticeable discrepancies occur near the extinction of the oscillations, at high blowing pressure.

\section{Introduction}

Reed instrument models are strongly nonlinear, which 22 explains how different oscillating regimes can be pro${ }_{23}$ duced for the same fingering. The produced regimes depend on the control parameters imposed by the musician, such as the blowing pressure or the opening at rest between the reed and the mouthpiece lay. This work assesses a choice done in many analytical studies of reed instrument models: the impact between the reed and the mouthpiece lay is ignored. In this simplification, called "ghost reed" hereafter, the reed penetrates the mouthpiece wall freely. This free interpenetration is nonphysical but it allows analytical developments [1,2] and numerical studies of minimal models $[3,4]$. To lesser extent, it may also lessen the computational cost. It is interesting to note that the "ghost reed" simplification is carried out de facto in models ignoring reed dynamics, such as in [5, 4, 19]. Lumped models of the contact between reed and lay have been developed using variable stiffness for the reed [6], or a separate contact force with stiffness and damping $[7,8]$. The last cited contact model was used in comparison with experimental data to estimate reed parameters [9]. In this paper, we investigate how the reed impact affects the overall behavior of the instrument model and which phenomena are reproduced similarly with and without the ghost reed simplification. This paper compares a recent lumped impact model [7] with the ghost reed model, in the case of a saxophone, using continuation associated with the harmonic balance method to describe the oscillatory regimes corresponding to the first two registers for the whole playing range with respect to the blowing pressure.

\section{Mathematical model of reed instrument}

The model studied here is similar to the one presented in [10] in the case of the clarinet. It is assumed that the acoustics of the resonator is linear. The relationship between the air flow $U$ and the acoustic pressure $P$ at the input of the resonator is classically written in the frequency domain thanks to the input impedance $Z(\omega)$, defined by:

$$
Z(\omega)=Z_{c} \frac{P(\omega)}{U(\omega)}=Z_{c} \sum_{n=0}^{+N_{m}} \frac{C_{n}}{i \omega-s_{n}}+\frac{\bar{C}_{n}}{i \omega-\bar{s}_{n}}
$$

where $Z_{c}$ is a characteristic impedance, $s_{n}$ are the (complex) poles of the impedance and $C_{n}$ the associated dimensionless residues. $N_{m}$ is taken equal to 8 in our case, in order to limit the complexity of the problem while representing all the main modes of the resonator. In the time domain, this relation gives the complex linear equations of the resonator model:

$$
\dot{p}_{n}(t)-s_{n} p_{n}(t)=Z_{c} C_{n} u(t),
$$

where $p_{n}$ are the modal pressures such that the total acoustic pressure is: $p(t)=2 \sum_{n=1}^{N_{m}} \Re\left(p_{n}(t)\right)$.

Following the work of [11], the input flow $u$ writes

$$
u=\zeta \operatorname{sign}(\gamma-p) \sqrt{|\gamma-p|} \max (x+1,0),
$$


where $\zeta$ is a dimensionless parameter characterizing the embouchure [10], $\gamma=p_{m} / p_{M}$ is the dimensionless version of the blowing pressure $p_{m}$, normalized by the static pressure $p_{M}$ necessary to displace the reed until the mouthpiece lay, and $p$ is the dimensionless acoustic pressure in the mouthpiece. $x$ is the dimensionless reed displacement, and the factor $\max (x+1,0)=\frac{1}{2}((x+1)+|x+1|)$ is equal to $x+1$ if the reed channel is open $(x+1>0)$ and 0 if it is closed $(x+1<0)$. Note that equation (3) is used for both cases studied: with and without the ghost reed simplification.

In order to facilitate numerical application of the harmonic balance and continuation methods, the absolute values in Eq. (3) are regularized with $|z| \simeq$ $\sqrt{z^{2}+\eta}$. One has $\operatorname{sign}(\gamma-p)=\frac{\gamma-p}{|\gamma-p|}$. The raw and regularized nonlinear characteristic are displayed in figure 1. The regularization parameter $\eta$ is taken equal to $10^{-3}$ so that the regularized nonlinear characteristic stays close to its raw version.

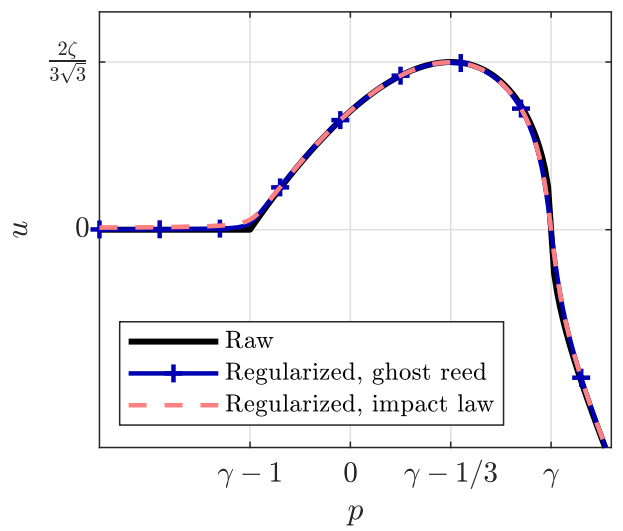

Figure 1: Nonlinear characteristics in static regimes: raw (Eq. (3)) and regularized versions, using $|z| \simeq \sqrt{z^{2}+\eta}$ with $\eta=10^{-3}$.

The motion of the reed is modeled by a one degree of freedom oscillator subject to a force coming from a pressure difference across the reed and an impact force. The dimensionless equation describing the motion is:

$$
\frac{\ddot{x}}{\omega_{r}^{2}}+q_{r} \frac{\dot{x}}{\omega_{r}}+x=-(\gamma-p)+F_{c}(x)-\beta \dot{x} F_{c}(x)
$$

where $\omega_{r}$ is the natural angular frequency of the reed and $q_{r}$ is a damping constant (inverse of a quality factor). $F_{c}$ follows the law suggested by [7] which is

$$
F_{c}(x)=-K_{c} \min (x+1,0)^{\alpha}
$$

where $K_{c}$ is the impact stiffness and $\alpha$ is an exponent which characterizes the impact. The term $\beta \dot{x} F_{c}$ can be thought of as a nonlinear damping term, i.e. the impact induces some loss of energy. It is regularized by writing $\min (x+1,0)=-\frac{1}{2}(|x+1|-(x+1))$ with the absolute value approximated as: $|x+1| \simeq$ $\sqrt{(x+1)^{2}+\eta}$. Considerations on the reed material and the mouthpiece material suggest an impact stiffness value of $K_{c}=100$, although there is no obvious experimental evidence. This means that the mouthpiece is one hundred times stiffer than the reed. The studies $[12,13]$ treat this question in more details. The ghost reed simplification is implemented by $K_{c}=0$ : the reed moves freely through the mouthpiece lay. All the values of the parameters are summarized in table 1.

\begin{tabular}{|l|c|c|}
\hline Parameter & Notation & Value \\
\hline \hline Impact stiffness & $K_{c}$ & 100 or 0 \\
\hline Impact exponent & $\alpha$ & 2 \\
\hline Impact damping & $\beta$ & 0.01 \\
\hline \hline Reed angular frequency & $\omega_{r}$ & 4224 rad.s $^{-1}$ \\
\hline Reed damping & $q_{r}$ & 1 \\
\hline \hline Blowing pressure & $\gamma$ & [variable] \\
\hline Embouchure & $\zeta$ & 0.6 \\
\hline \hline Regularization & $\eta$ & 0.001 \\
\hline
\end{tabular}

Table 1: Parameters of the model. Parameters with no units are dimensionless.

\section{Comparison between the ghost reed simplification and the impact model}

Now the comparison is made between the ghost reed simplification, without impact force to limit the reed displacement $\left(K_{c}=0\right)$, and the model with impact $\left(K_{c}>0\right.$, here $\left.K_{c}=100\right)$. The modal parameters of the resonator are deduced from the impedance measured on an alto saxophone. The fingering of a low $\mathrm{D} \sharp$ is used. This fingering is the sixth lowest of the saxophone, and it exhibits both first and second register regimes. Higher fingerings produce less or none of the second register, and lower fingerings produce a great variety of regimes that make the analysis of the bifurcation diagram tedious.

Periodic solutions of the models are found using the harmonic balance method, where all the variables are decomposed into a Fourier series up to harmonics $H$ (see for example [14]). Asymptotic numerical method (ANM) is used to produce the bifurcation diagram corresponding to each model $[15,16]$. In this work, $H=50$ for first register regimes and $H=80$ for second register regimes. Only periodic regimes can be computed by the harmonic balance method, so the waveform comparison excludes transients. Stability of the regimes is determined using the method presented in [17] and [18]. 


\subsection{Waveforms}

As a first comparison step, temporal waveforms of the reed displacement $x$ as well as the acoustic pressure $p$ and flow $u$, are displayed in figures 2 and 3 . These regimes correspond to the first register of the instrument: their frequency is close to the first resonance frequency of the resonator. Figure 2 shows the established periodic regime obtained for $\gamma=0.5$ : it corresponds to the standard Helmholtz motion, typical of conical instruments [19]. As expected, the reed displacement waveforms are quite different: the impact force penalizes the displacement of the reed below $x=-1$, whereas the ghost reed simplification lets it move freely below $x=-1$. Note that this formulation of impact force lets the reed squash against the lay and go slightly below -1 , though far less than the ghost reed model. This is the main difference between the reed displacements generated by the two models. Over the period, the displacement signals are most similar right before the contact occurs. As for the acoustic pressure $p$, the two waveforms are nearly superimposed, except for some higher frequency components. This is confirmed by a good match in the amplitude of the harmonics, especially the first nine. Similar comments can be made on the flow $u$. For this particular value of the blowing pressure $\gamma$, the reed impact model has no major influence on the pressure and flow waveform.
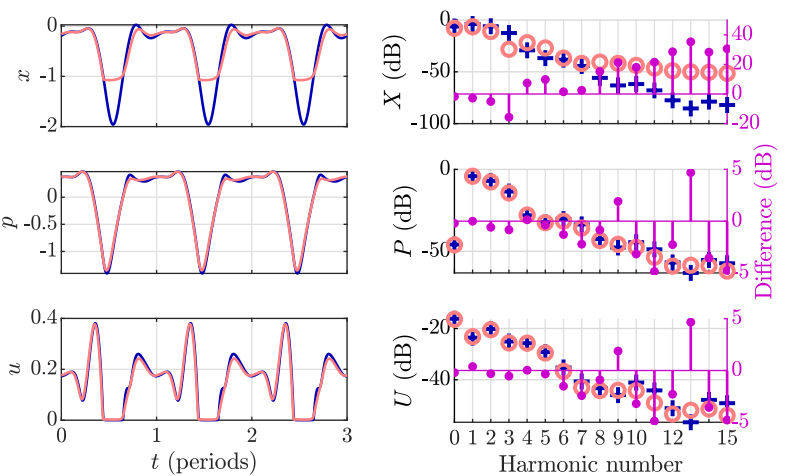

Figure 2: Steady periodic regime for the reed displacement $x$, and the acoustic pressure $p$ and flow $u$. Waveforms (left) and amplitude of harmonics (right). Model with impact force (light red circles) and ghost reed simplification (dark blue pluses), difference in harmonic amplitude between models (purple dots). Blowing pressure $\gamma=0.5$.

Figure 3 presents the waveforms obtained for a higher value of the blowing pressure $\gamma=1.5$. Eventhough $\gamma>1$ corresponds to sufficient pressure to close the reed channel completely in a static configuration, oscillations may still be sustained for $\gamma>1$. The obtained oscillating regime correspond to the inverted Helmholtz motion [19], where the reed stays in contact with the lay for more than half the period. In this case, the reed displacement waveform presents even more differences than on figure 2. However, the acoustic pressure and flow waveforms stay very similar, as they are on figure 2, and their first seven harmonics are very close. In order to qualify the effect of the reed impact model on a variety of regimes, the associated bifurcation diagrams are now studied.
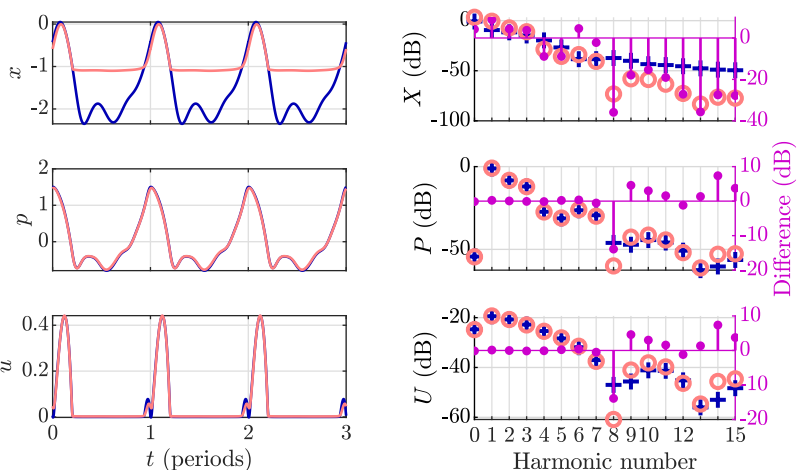

Figure 3: Steady periodic regime for acoustic pressure $p$ and flow $u$, and reed displacement $x$. Waveforms (left) and amplitude of harmonics (right). Model with impact force (light red circles) and ghost reed simplification (dark blue pluses), difference in harmonic amplitude between models (purple dots). Blowing pressure $\gamma=1.5$.

\subsection{Bifurcation diagrams}

The bifurcation diagram in figure 4 shows the $\mathrm{L}^{2}$ norm of the acoustic pressure $p$, with respect to the blowing pressure $\gamma$. The $\mathrm{L}^{2}$ norm is defined by

$$
\|p\|_{L^{2}}=\frac{1}{T} \int_{0}^{T} p(t)^{2} \mathrm{~d} t,
$$

where $T$ is the period of $p$. The first register is computed with Fourier series truncated at $H=50$ harmonics, the second one with $H=80$ harmonics. These truncation orders have been chosen to ensure a good convergence of the solution and of the stability information of the periodic solution branches. Several common features appear between the structure of the overlaid diagrams, corresponding to the ghost reed and to the impact model. In terms of oscillation threshold, both cases exhibit two Hopf bifurcations on the equilibrium branch (not shown here, corresponds to $\|p\|_{L^{2}}=0$ ). They are marked $\mathrm{H}$ in figure 4. The first one, a sub-critical Hopf bifurcation around $\gamma=0.4$ for both models, is associated with the first register of the saxophone. The second one, a super-critical Hopf bifurcation around $\gamma=0.43$ for both models as well, is associated with the second register. Branches of the two models share common characteristics, but are not superimposed. For instance, the range of $\gamma$ where stable periodic oscillations exist is reduced when taking into account the impact force. The right Neimark-Sacker bifurcation on the 
second register solution branch appears very sensible to the impact model in this case. Without impact force, it is located around $\gamma=1.25$, below the fold bifurcation point. However, with impact force, the right Neimarck-Sacker bifurcation is located around $\gamma=1.8$, above the fold bifurcation. The highest values of the blowing pressure present the most discrepancies between the two models. This can be explained by the fact the duration of the contact between the reed and the lay is longer for higher blowing pressure values, and thus it is the region where the impact model has the most influence.

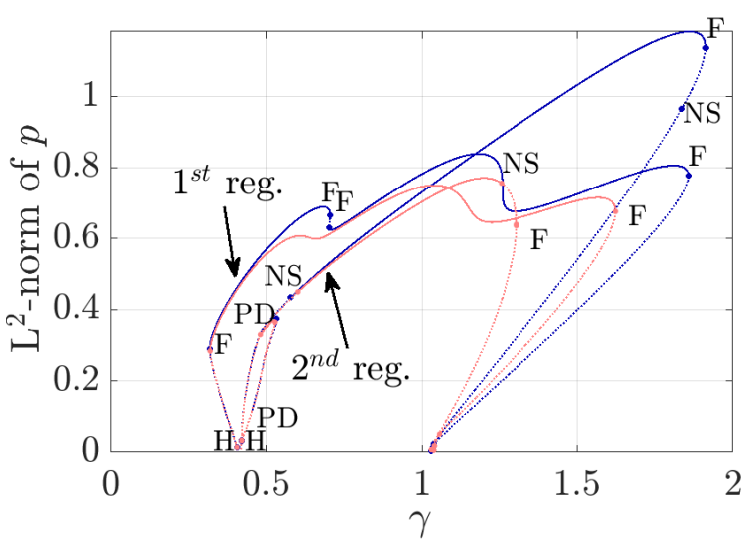

Figure 4: Bifurcation diagram: $\mathrm{L}^{2}$ norm of the acoustic pressure $p$ with respect to blowing pressure $\gamma$. Stable solutions are in solid lines, unstable solutions are in dotted lines. Dark blue: ghost reed simplification; light red: impact force model. Bifurcation labels are H: Hopf; PD: period doubling; F: fold; NS: NeimarkSacker.
Figure 5 is a close-up of figure 4 for values of $\gamma$ smaller than 1. In this area, which is expected to be more commonly reached than the area $\gamma>1$ by the instrument players (it corresponds to moderate playing levels), the differences between the two models exist but are mostly negligible. The periodic solutions arising from the two Hopf bifurcations encounter generic bifurcations of periodic solutions, namely fold bifurcation, period doubling bifurcation and NeimarkSacker bifurcation. The bifurcations are almost superimposed for both models. The branch of unstable periodic solutions arising from the period doubling bifurcations are exactly superimposed. Despite a very good agreement over the range of the figure 5 there is a non-negligible difference on the first register for $0.5<\gamma<0.7$, with two fold bifurcations for the ghost reed simplification that are not found with the impact model.

The same study has been performed for other values of $\zeta$, which confirms the results presented here. With the formulation of the impact chosen in this paper, it is possible to vary continuously between impact model and ghost reed simplifications: for values of $K_{c}$ in between 0 and 100, the solution branches are in between the two branches displayed in figure 4 .

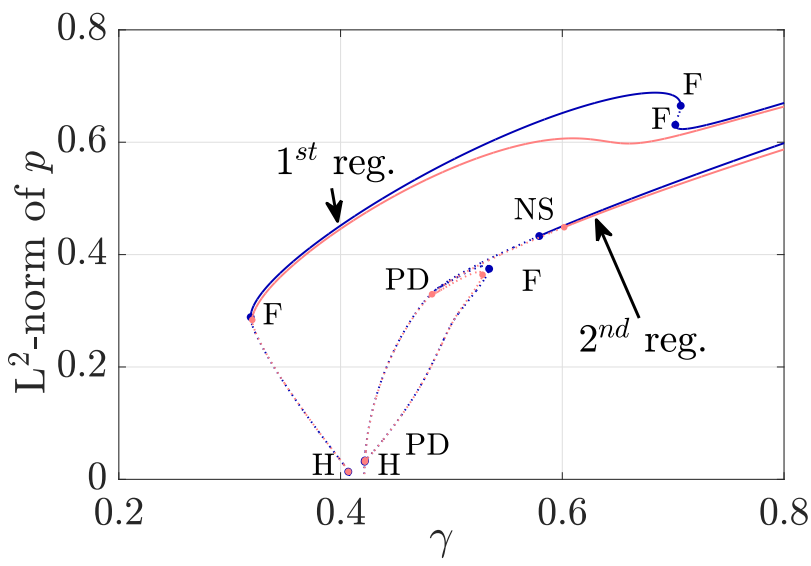

Figure 5: Zoom of figure 4.

\section{Conclusion}

Taking into account reed impact in a saxophone model has definite influence on regimes obtained with high values of the blowing pressure parameter, for which the reed is in contact with the mouthpiece lay for a large part of the oscillation period. However, if the study is limited at low blowing pressures, around the low oscillation threshold for instance, the ghost reed simplification delivers results very close to elaborate impact models at a much lower computational cost. The effect of the nature of the reed impact on the transients and other non-stationary phenomena remains to be studied, as part of a much wider field of investigation.

\section{Acknowledgments}

This work has been carried out in the framework of the Labex MEC (ANR-10-LABEX-0092) and of the A*MIDEX project (ANR-11-IDEX-0001-02), funded by the Investissements d'Avenir French Government program managed by the French National Research Agency (ANR). This study has been supported by the French ANR 659 LabCom LIAMFI (ANR-16-LCV2007-01).

\section{References}

[1] J.-P. Dalmont, J. Gilbert, and J. Kergomard, "Reed instruments, from small to large amplitude periodic oscillations and the helmholtz motion analogy," Acta Acustica united with Acustica, vol. 86, no. 4, pp. 671-684, 2000.

[2] C. Nederveen, Acoustical aspects of musical instruments. Northern Illinois Univ. Press, 1998. 
[3] J.-B. Doc, C. Vergez, and S. Missoum, "A minimal model of a single-reed instrument producing quasi-periodic sounds," Acta Acustica united with Acustica, vol. 100, no. 3, pp. 543-554, 2014.

[4] J. Kergomard, P. Guillemain, F. Silva, and S. Karkar, "Idealized digital models for conical reed instruments, with focus on the internal pressure waveform," The Journal of the Acoustical Society of America, vol. 139, no. 2, pp. 927-937, 2016.

[5] A. Guilloteau, P. Guillemain, J. Kergomard, and M. Jousserand, "The effect of the size of the opening on the acoustic power radiated by a reed woodwind instrument," Journal of Sound and Vibration, vol. 343, pp. 166-175, 2015.

[6] M. Van Walstijn and F. Avanzini, "Modelling the mechanical response of the reed-mouthpiecelip system of a clarinet. part ii: A lumped model approximation," Acta Acustica united with Acustica, vol. 93, no. 3, pp. 435-446, 2007.

[7] S. Bilbao, A. Torin, and V. Chatziioannou, "Numerical modeling of collisions in musical instruments," Acta Acustica united with Acustica, vol. 101, no. 1, pp. 155-173, 2015.

[8] V. Chatziioannou and M. van Walstijn, "Estimation of clarinet reed parameters by inverse modelling," Acta Acustica united with Acustica, vol. 98, no. 4, pp. 629-639, 2012.

[9] A. Muñoz Arancón, B. Gazengel, J.-P. Dalmont, and E. Conan, "Estimation of saxophone reed parameters during playing," The Journal of the Acoustical Society of America, vol. 139, no. 5, pp. 2754-2765, 2016.

[10] W. L. Coyle, P. Guillemain, J. Kergomard, and J.-P. Dalmont, "Predicting playing frequencies for clarinets: A comparison between numerical simulations and simplified analytical formulas," The Journal of the Acoustical Society of America, vol. 138, no. 5, pp. 2770-2781, 2015.

[11] T. A. Wilson and G. S. Beavers, "Operating modes of the clarinet," The Journal of the Acoustical Society of America, vol. 56, no. 2, pp. 653-658, 1974.

[12] J.-P. Dalmont, J. Gilbert, and S. Ollivier, "Nonlinear characteristics of single-reed instruments: Quasistatic volume flow and reed opening measurements," The Journal of the Acoustical Society of America, vol. 114, no. 4, pp. 2253$2262,2003$.

[13] F. Avanzini and M. van Walstijn, "Modelling the mechanical response of the reed-mouthpiece-lip system of a clarinet. part i. a one-dimensional distributed model," Acta Acustica united with 307 Acustica, vol. 90, no. 3, pp. 537-547, 2004.

[14] J. Gilbert, J. Kergomard, and E. Ngoya, "Calculation of the steady-state oscillations of a clarinet using the harmonic balance technique," The journal of the Acoustical Society of America, vol. 86, no. 1, pp. 35-41, 1989.

[15] B. Cochelin and C. Vergez, "A high order purely frequency-based harmonic balance formulation for continuation of periodic solutions," Journal of sound and vibration, vol. 324 , no. $1-2 \overline{\text { pp. } 243-}$ $262,2009$.

[16] L. Guillot, B. Cochelin, and C. Vergez, "A taylor series-based continuation method for solutions of dynamical systems," Nonlinear Dynamics, pp. 119, 2019.

[17] A. Lazarus and O. Thomas, "A harmonic-based method for computing the stability of periodic solutions of dynamical systems," Comptes Rendus Mécanique, vol. 338, no. 9, pp. $\overline{510-517}$, 2010.

[18] B. Bentvelsen and A. Lazarus, "Modal and stability analysis of structures in periodic elastic states: application to the ziegler column," Nonlinear Dynamics, vol. 91, no. 2, pp. 1349 1370, 2018.

[19] S. Ollivier, J.-P. Dalmont, and J. Kergomard, "Idealized models of reed woodwinds. part i: Analogy with the bowed string," Acta acustica united with acustica, vol. 90, no. 6, pp. 11921203, 2004. 